\title{
Design and experimental study of 110kV Substation Transformer active noise control system
}

\author{
J.W. Wang, L.M. YING, P. Yang, Y.T. Jia, L. Yang \\ School of Electrical Engineering Wuhan University, Wuhan, Wuhan, China \\ Q. Liu \\ Wuhan Electric Power Technical College, Wuhan, China
}

\begin{abstract}
An active noise control system of power transformer is designed and implemented in this paper. EL-VB-FxLMS algorithm is used in the system as the control algorithm, the performance of which is analyzed and compared to BFxLMS algorithm. Based on EL-VB-FxLMS algorithm, a set of practice ANC system is built by industrial PC, PCI data card, microphone and loudspeaker. Finally, an experiment was carried out in a sub-station.
\end{abstract}

KEYWORD: Transformer noise; Active noise control; EL-VB-FxLMS algorithm; experiment

\section{INTRODUCTION}

With the rapid development of economy and urbanization, noise nuisance problem of power transformer grows increasingly serious. More and more substations of urban distribution network are adjacent to the residential community. Traditional passive noise reduction method is not only expensive, but also poor control effect of low frequency noise. The proposal of active noise control (ANC) technology provides a new way for controlling low-frequency noise $^{1}$. The basic idea of the technology is to track the noise signal, and send secondary sound having opposite phase and same amplitude with the noise for destructive interference in the spatial sound field to reduce the noise. An active noise control system consists of two parts, the controller and the electroacoustic device. The controller is used to track the noise signal, while the electro-acoustic device is to pick up the noise signal and output the secondary sound. Among them, the performance of control algorithm ${ }^{2}$ and the layout scheme of secondary sound source are the key factors in determining the noise reduction effect.

\section{CONTROL ALGORITHM DESIGN}

\subsection{A variable block length filtering LMS algorithm with error limit}

In this work, a variable block length filtering LMS (EL-VB-FxLMS) algorithm with error limit is used. Based on FXLMS, the reference signal is processed by block, and a block of data is calculated by parallel computing. The accumulated error and the error limit are introduced as the discriminative conditions of changing block length to reduce the steady-state error of the algorithm and increase the stability of the system. Algorithm principle is as follows:

The reference signal is supposed as:

$\mathbf{x}(n)=[x(n), x(n-1), \ldots, x(n-M+1)]^{T}$

$\mathrm{M}$ is the length of the filter, $\mathrm{k}$ is the number of the block, L is the length of the data, then the relationship between $\mathrm{n}$ and $\mathrm{k}$ can be expressed as:

$n=k L+i \quad i=0,1, \ldots, L-1$

The data of block $k$ can be expressed as ${ }^{4}$ :

$\mathbf{x}_{k}(L)=[x(k L), x(k L+1), \cdots, x(k L+L-1)]^{T}$

After block processing, the reference signal can be written as:

$\mathbf{x}(n)=\left[\mathbf{x}_{1}(k L), \mathbf{x}_{2}(k L-1), \ldots, \mathbf{x}_{k}(k L-M+1)\right]^{T}$

The output signal can be represented as:

$$
\begin{aligned}
y(n) & =\mathbf{w}^{H}(n) \mathbf{x}(n) \\
& =\mathbf{w}^{H}(k) \mathbf{x}(k L+i)
\end{aligned}
$$

The error signal can be represented as:

$$
\begin{aligned}
e(n) & =d(n)-y(n) \\
& =d(k L+i)-y(k L+i)
\end{aligned}
$$

The weights update formula can be represented as:

$\mathbf{w}(n)=\mathbf{w}(k)+\mu \sum_{i=0}^{L-1} \mathbf{x}(k L+i) e(k L+i) \quad i=0,1, \ldots, L-1$ 
$\overline{\mathbf{x}(k)}$ and $\overline{e(k)}$ stands for the mean value of the input signal and the error signal in the duration of the block K respectively, the update formula of the weight can be rewritten as:

$$
\mathbf{w}(k+1)=\mathbf{w}(k)+L \mu \overline{\mathbf{x}(k) e(k)}
$$

It can be seen that the block length $L$ in the VBFxLMS algorithm plays the similar role with the step factor $\mu$ in the LMS algorithm. In order to control the error mean $\bar{e}$, we can control the update of the weight vector by changing the block length $L$. The algorithm is equivalent to the FxLMS algorithm when the block length $L$ equals 1, while the system will be unstable when the block length is too large. The error limit aims at controlling the length of the block for achieving the optimal performance.

$E$ is the accumulated error which is defined as the sum of the absolute values of the product between input signal $x(n)$ and the error signal $e(n)$.

$$
E=\sum_{i=0}^{n}|E(i)|=\sum_{i=0}^{n}|x(i) e(i)|
$$

$E$ represents the accumulated error of the system since the last update of weight vector. E increases with the algorithm process, its value and growth speed reflect the degree of interference and stability. Setting the error limit as $E_{\max }$, when the error accumulates to a certain extent and exceeds the error limit, and then update the weight vector. The update process of weight vector is shown in Figure 1.

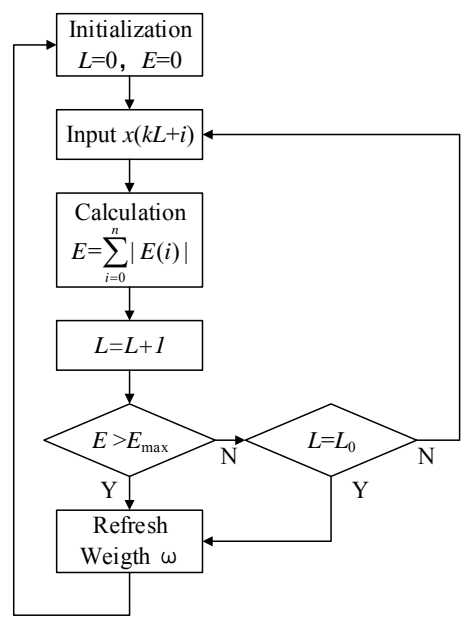

Figure 1. The update process of Weight vector

\subsection{Algorithm simulation}

In order to verify the performance of the algorithm, the EL-VB-FxLMS algorithm is simulated by using the measured noise signal of transformer in one substation. The schematic diagram of the control system is shown in Figure 2.

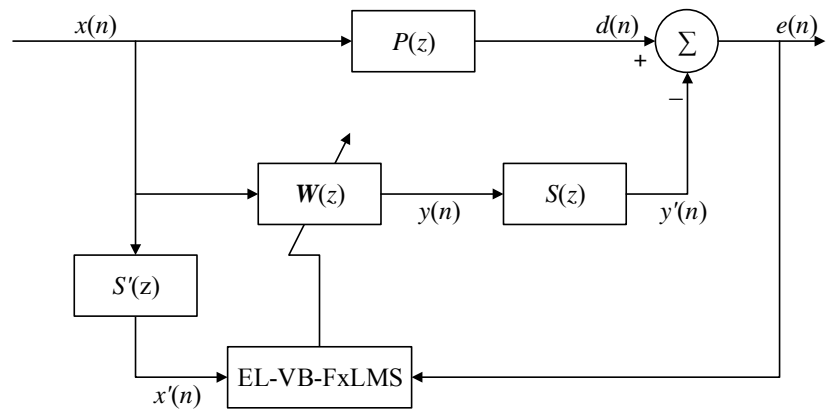

Figure 2. Transformer noise ANC system using EL-VBFxLMS algorithm block diagram.

$P(z)$ is the primary channel transfer function of the noise source to the sensor, $S(z)$ is the secondary channel transfer function of the filter output signal to the desired signal ${ }^{3}$.

Set $P(z)=[0.01,0.25,0.5,0.25,0.01], S(z)=0.25 \mathrm{P}(z)$. The sampling frequency $f_{s}$ is $25000 \mathrm{~Hz}$, the filter order $M$ is 16 ; the step $\mu$ is 0.03 , the update block length $L_{0}$ is 16 ; and the error limit $E_{\max }$ is 1 . In contrast, the BFxLMS algorithm is simulated with the same parameters, and the results are shown in Figure 3. Wherein Figure 3(a) is algorithm simulation results of this paper, Figure 3(b) is BFxLMS algorithm simulation results.

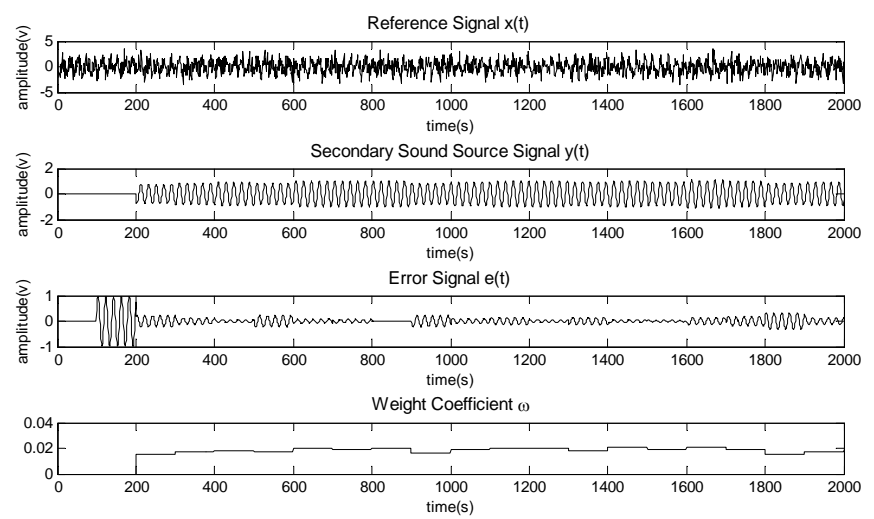

(a)

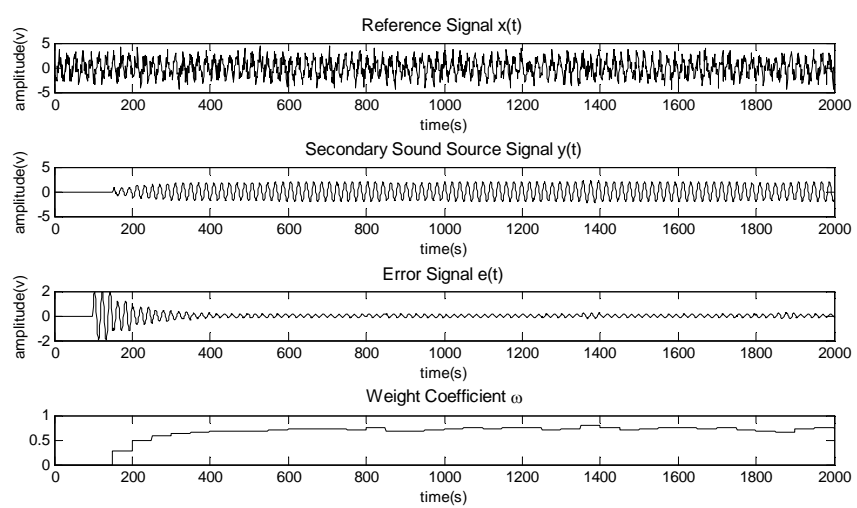

(b)

Figure 3. Diagram of Simulation results using different ANC algorithm.(a)BFxLMS;(b)EL-VB-FxLMS.

Analysis results state that the algorithm in this work has faster convergence speed than the BFxLMS algorithm. In addition, it has reliable per- 
formances with small steady-state error and don't exist unstable phenomenons of the BFxLMS. Thus, it's worthy of further development and research.

\section{SYSTEM TESTING PRACTICE}

\subsection{Controller hardware design (components)}

Taking the existence of a strong magnetic in substation into account, electromagnetic compatibility and Industrial Personal Computer(IPC) are used as the hardware carrier of the controller. IPC which has the multi-threading processing ability and powerful computing performance, is able to ensure the good delay performance of the algorithm. At the same time, its rich function expansion slot can pick powerful data acquisition card according to different application environment. In this paper, we use PCI multi-function data acquisition card(DAC) and data output card(DOC) to achieve data input and output and $\mathrm{D} / \mathrm{A}, \mathrm{A} / \mathrm{D}$ function. We using $\mathrm{C}++$ to build the control system software with interactive interface, which can start and stop system, analysing the noise spectrum and displaying the real-time noise waveform. The photos of Controller hardware and software are shown in Figure 4.

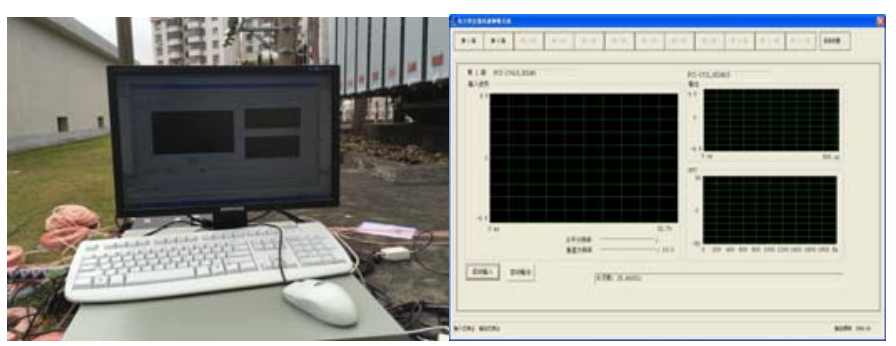

Figure 4. Controller software and hardware photos.

\subsection{The design of electro acoustic devices}

Repolarized test capacitor microphone and preamplifier are used as reference sensor and error sensor to record the initial and remnant noise after noise reduction processing of the transformer, respectively. The frequency range is $6.3-20 \mathrm{kHz}$, and the sensitivity is $50 \mathrm{mv} / \mathrm{pa}$, which can meet the requirements of low frequency noise recording. HiVi loudspeaker which can bear large load power and has good low frequency response is used as secondary sound source array. It can output the secondary sound power continuously. The secondary sound source array device and the sensor are shown in Figure 5. The coaxial cable connection is adopted between the sensor and the industrial control computer data acquisition card to reduce the influence of the electromagnetic environment of the transformer substation. ANC system wiring diagram is shown in Figure 6.

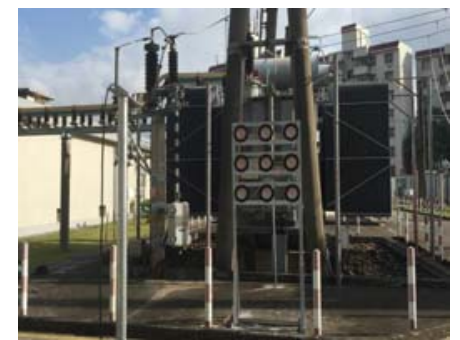

(a)

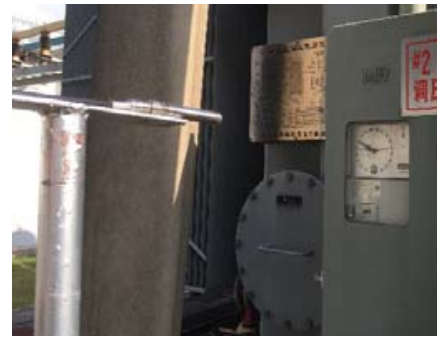

(b)
Figure 5. Schematic diagram of electro acoustic devices. (a)Secondary sound source array; (b) Microphone.

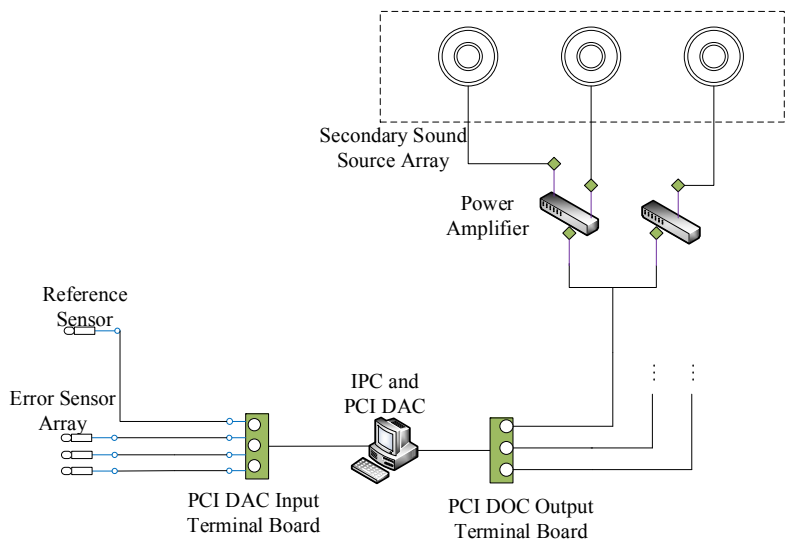

Figure 6. Schematic diagram of ANC System Wiring.

\subsection{Test experiment in $110 \mathrm{kV}$ substation}

Cooperating the software and hardware above, an active noise control system for power transformer based on EL-VB-FxLMS algorithm is installed in actual substation. Nine speakers are form a $3 \times 3 \mathrm{sec}-$ ondary sound source array. The lateral and longitudinal spacing of each speaker both are $0.3 \mathrm{~m}$ and the minimum distance from the ground is $1.5 \mathrm{~m}$. A speaker is used as reference sensor. The distance from the ground is $1.7 \mathrm{~m}$ and the distance from the noise source is $0.4 \mathrm{~m}$. Another three speakers are form error sensor array. The distance from the ground is $1.7 \mathrm{~m}$ and the distance from the noise source is $7 \mathrm{~m}$. Noise before and after noise reduction were recorded by a noise meter. Equipment placement of the active noise control system is shown in Figure 7. Schematic diagram of measuring point distribution is shown in Figure 8.

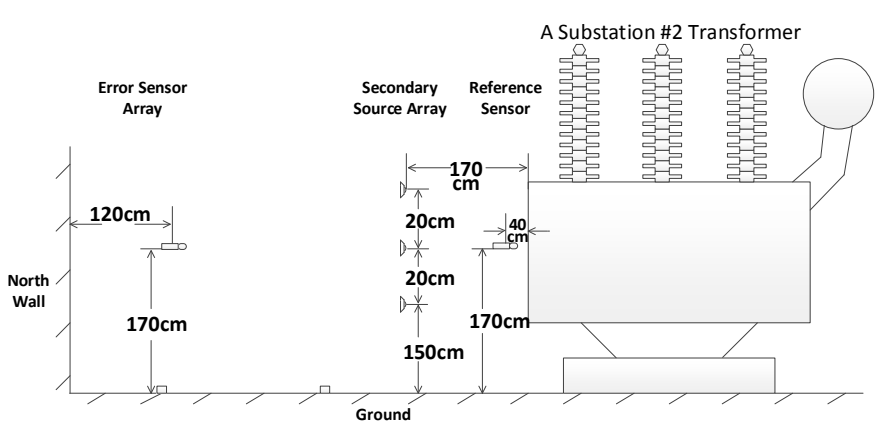

Figure 7. Electroacoustic device laying scheme diagram. 


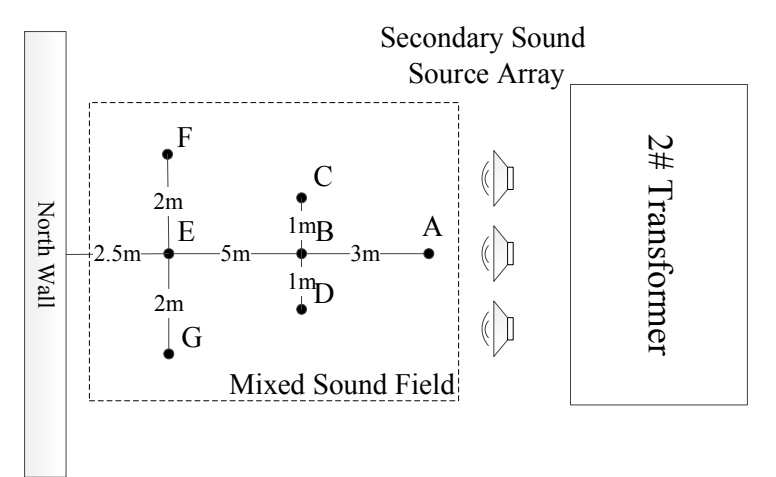

Figure 8. Schematic diagram of measuring point distribution.

Due to the relatively quiet environment at noon, less interference factors, measured data at noon. The measured data have credibility. During installation and commissioning, Active noise control system runs well, and no abnormal termination and error being given phenomenon. Electro-acoustic devices and controller hardware running stable, with no abnormal fever and failure phenomena. Since the main energy of the transformer noise is concentrated in a few single frequency noise ${ }^{6}$, such as $100 \mathrm{~Hz}, 200 \mathrm{~Hz}$, $300 \mathrm{~Hz}$, so the test were also recorded three singlefrequency noise. Measurement results shown in Figure 9.

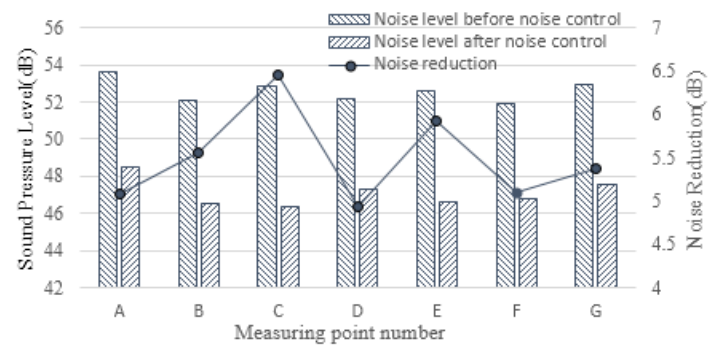

(a) Total noise

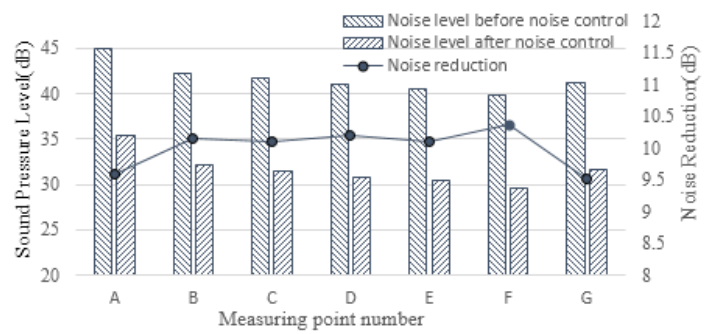

(b) $100 \mathrm{~Hz}$ single frequency noise

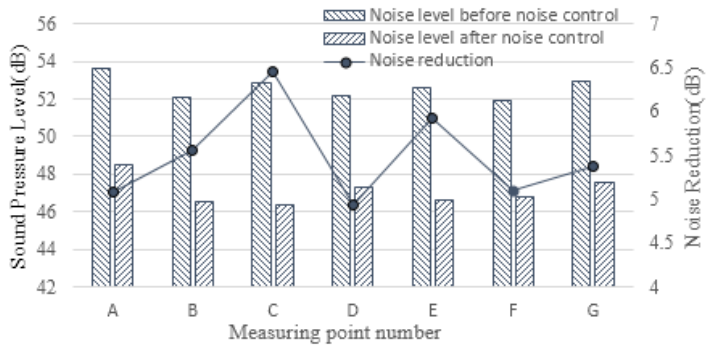

(c) $200 \mathrm{~Hz}$ single frequency noise

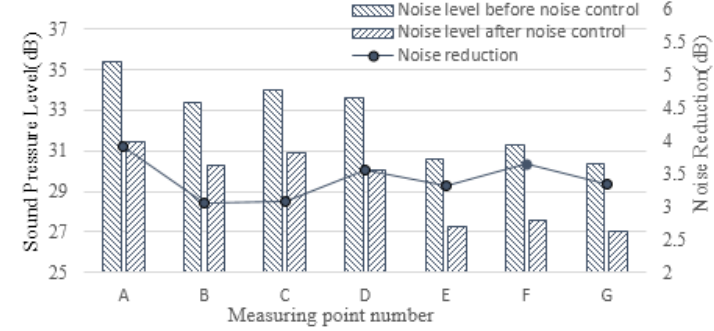

(d) $300 \mathrm{~Hz}$ single frequency noise.

Figure 9. Experimental results analysis.

From the results, it can be seen that the ANC system designed in this paper can obtain the average $5.5 \mathrm{~dB}$ noise reduction in a certain area. The noise reduction effect of $100 \mathrm{~Hz}, 200 \mathrm{~Hz}, 300 \mathrm{~Hz}$ component is better, which proves the validity of the ANC to the low frequency noise.

\section{CONCLUSIONS}

The paper proposes to use a variable block length filtering LMS algorithm with error limit to active control of transformer noise. Simulation results show that the proposed algorithm has better performance than the BFxLMS algorithm. The paper use industrial control computer, PCI data card, and $\mathrm{C}++$ programming are used to achieve the function of ANC system controller, and use microphone and loudspeaker are used to constitute the electroacoustic devices. The experimental results show that the ANC system designed in this paper can run stably, and achieve the effect of reducing the noise of $5.5 \mathrm{~dB}$, which provides experience and guidance for the next phase of practical research.

\section{REFERENCES}

H.F. Zhao, et al. 2015. Research on Substation Noise and Design of the Control System. Power System and Clean Energy 31(10):55-60.

L.M. Zhang, et al. 2012. Active Control of Transformer Noise with an Internally Synthesized Reference Signal. Journal of Sound and Vibration 331:3466-3475.

P. Lueg. 1936. Process of Silencing Sound Oscillations.US Patent: 2043416.

N.V. George \& G. Panda. 2013. Advances in Active Noise Control: A Survey, with Emphasis on Recent Nonlinear Techniques. Signal Processing 93: 363-377.

Y. Kajikawa, et al. 2012. Recent advances on active noise control: open issues and innovative applications. Transactions on Signal and Information Processing 1: 1-22.

Y. Zheng, et al. 2015. Variable block-size BLMS algorithm for active noise control of power transformer. Transformer 52(9):28-32. 